ISSN: 2773-7349

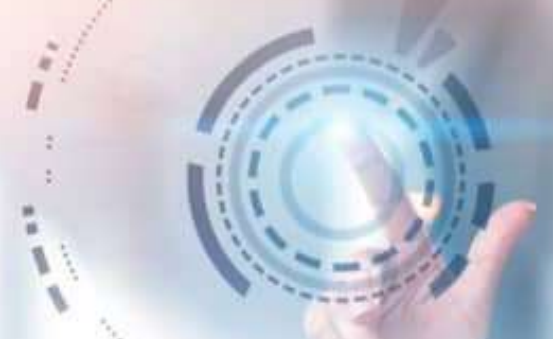

Sociedad \& Tecnología Revista del Instituto Tecnológico Superior Jubones

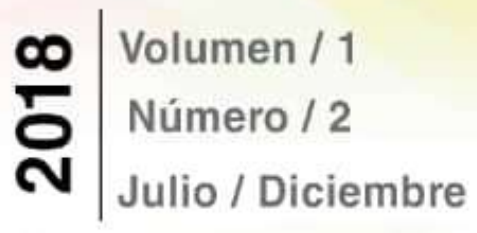

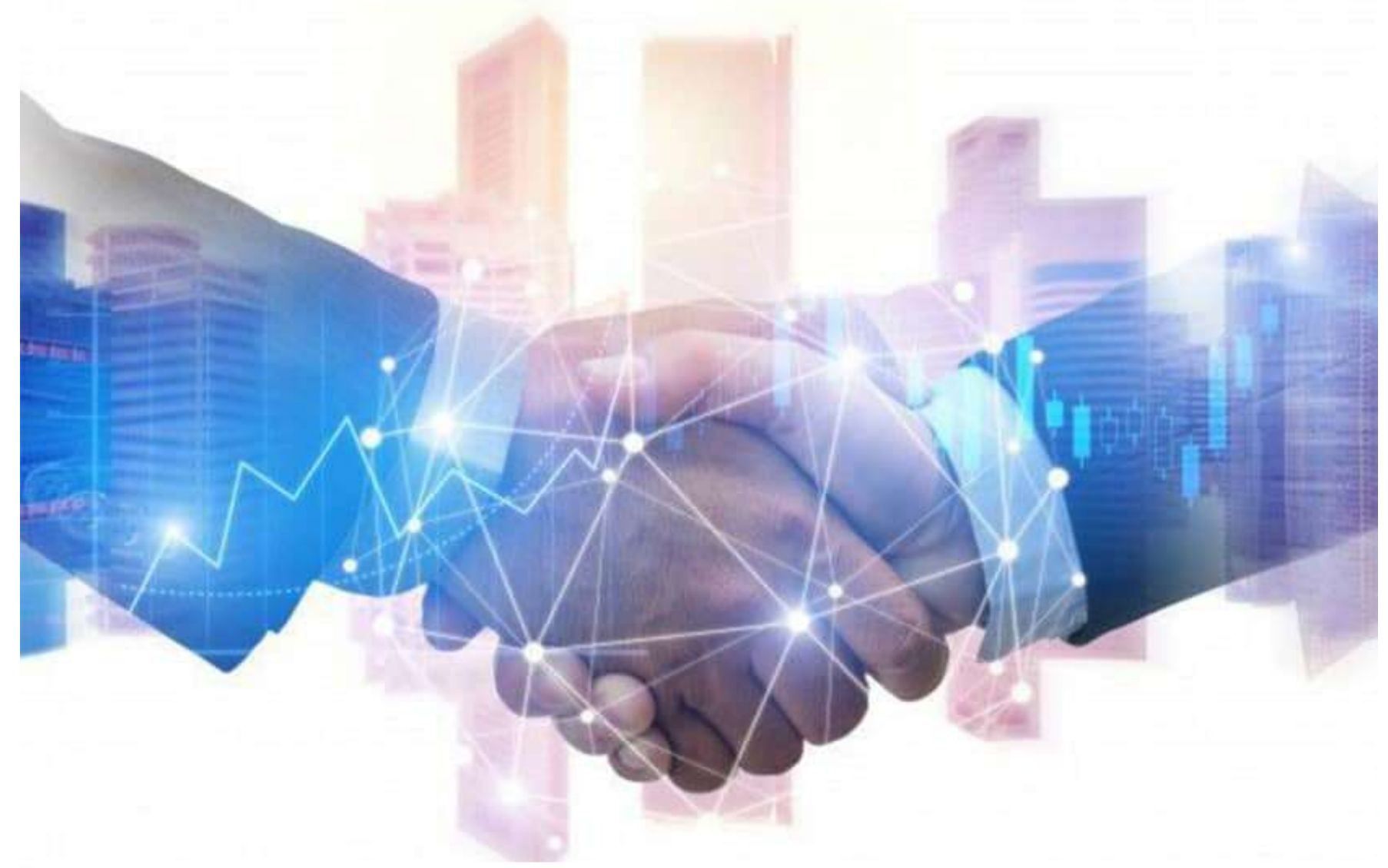




\title{
NUEVAS PERSPECTIVAS EN LA SUPERACIÓN DE BIBLIOTECARIOS DE LA UNIVERSIDAD DE ORIENTE.
}

EW PERSPECTIVES IN THE IMPROVEMENT OF LIBRARIANS OF THE UNIVERSIDAD DE ORIENTE.

\author{
Silvia Idania Ramírez Rodríguez \\ E-mail: silviarr@uo.edu.cu \\ ORCID: https://orcid.org/0000-0002-5636-7640 \\ Adia Gell Labañino, \\ E-mail: adiagell9@gmail.com \\ ORCID: https://orcid.org/0000-0002-3212-9672 \\ Aniuska Tito Durán \\ E-mail: aniuska.tito@uo.edu.cu \\ ORCID: https://orcid.org/0000-0002-4343-2140 \\ Universidad de Oriente, Santiago de Cuba, Cuba
}

Cita sugerida (APA, séptima edición)

Ramírez Rodríguez, S. I., Gell Labañino, A., \& Tito Durán, A. (2018). Nuevas perspectivas en la superación de bibliotecarios de la universidad de oriente. Revista Sociedad \& Tecnología, 1(2), 2-10.

\section{RESUMEN}

Las bibliotecas universitarias dirigen sus esfuerzos hacia el desarrollo y la formación de profesionales que la sociedad necesita, con sentido de su identidad nacional, identificado con su cultura y su nación socialista. En este sentido, es oportuno significar cómo al bibliotecario universitario le corresponde este encargo social y para el cual debe estar preparado, a fin de cumplir con eficacia y efectividad las funciones inherentes al cargo que desempeña. De ahí, que la presente investigación responda a la necesidad de aportar una estrategia de superación técnico- pedagógica sustentada en un modelo de igual naturaleza, debido a la existencia de insuficiencias en su preparación limitando su desempeño profesional. En la búsqueda de alternativas de solución para revertir la situación descrita, se revela la necesidad de fundamentar una propuesta teórica y práctica asociada a la formación permanente de los bibliotecarios de la Universidad de Oriente, a partir de comprender sus particularidades, necesidades, limitaciones, ventajas e intereses. El modelo es contentivo de: conceptos, caracterización, componentes y relaciones que lo fundamentan. La estrategia se organiza y estructura en tres etapas. La viabilidad y pertinencia de los aportes se corroboran a partir de métodos y técnicas como el criterio de especialistas y talleres de sensibilización y socialización.

\section{Palabras clave:}

Biblioteca universitaria, superación de bibliotecarios, estrategia de superación, modelo pedagógico.

\begin{abstract}
The university libraries direct their efforts towards the development and training of professionals that society needs, with a sense of their national identity, identified with their culture and their socialist nation. In this sense, it is opportune to mean how the university librarian is responsible for this social task and for which he must be prepared, in order to effectively and effectively fulfill the functions inherent to the position he holds. Hence, that this research responds to the need to provide a strategy of technicalpedagogical improvement supported by a model of
\end{abstract}


the same nature, due to the existence of inadequacies in their preparation limiting their professional performance. In the search for alternative solutions to reverse the situation described, it reveals the need to base a theoretical and practical proposal associated with the permanent training of librarians of the Universidad de Oriente, from understanding their particularities, needs, limitations, advantages and interests. The model is content of: concepts, characterization, components and relationships that support it. The strategy is organized and structured in three stages. The viability and relevance of the contributions are corroborated from methods and techniques such as the criteria of specialists and awareness and socialization workshops.

\section{Keywords:}

University library, overcoming librarians, improvement strategy, pedagogical model

\section{INTRODUCCIÓN}

El modelo actual de formación del profesional de la especialidad de Bibliotecología y Técnicas Documentarias presenta una dinámica que establece dos momentos fundamentales: el primero de carácter predominantemente académico y el segundo la organización de la práctica laboral con el propósito de la preparación teórica y práctica para enfrentarse a los cambios que se produzcan en las bibliotecas; sin embargo, aún no se logra la efectividad en su desempeño laboral.

Ello es consecuencia por la carencia del personal docente especializado que asume la docencia, por lo que en ocasiones las asignaturas de la especialidad son impartidas por docentes de otras especialidades, lo que deriva el cumplimiento parcial de los programas de estudio; dado por las insuficiencias de la planificación, sistematización y control de la práctica pre-profesional, así como la falta de recursos bibliográficos de la especialidad, entre otras.

Las exigencias sociales precisan de bibliotecarios comprometidos con la sociedad y su tiempo, con amplia cultura, sobre la base de una sólida formación de capacidades cognoscitivas y habilidades técnico profesionales, que le permitan enfrentar con éxito los disímiles problemas que se presentan en las escuelas, con expresión en la práctica pedagógica.

3
Con independencia al trabajo desarrollado, existe una serie de insuficiencias, que incide de forma negativa en la calidad de la formación y del funcionamiento de las bibliotecas universitarias, y que de manera empírica, se ha descubierto a través del análisis crítico de diversos documentos pertenecientes a distintos niveles de dirección, tales como: registros de ayuda metodológica, documentos del trabajo cotidiano, informes parciales y finales de etapas y cursos a lo que se le suma los estudios preliminares realizados por las autoras de esta investigación en las tesis de maestría y de doctorado relacionados al trabajo que se desarrolla, todo lo cual permite identificar como principales insuficiencias, las siguientes:

$>$ Escaso conocimiento de las normas y técnicas profesionales de los bibliotecarios universitarios manifestadas en su desempeño laboral.

> Carencias en la preparación pedagógica de los bibliotecarios que restringen la efectividad de su desempeño profesional, afectando la eficacia del proceso formativo en las bibliotecas universitarias.

$>$ Incongruente concepción del proceso de capacitación de los bibliotecarios a partir de las exigencias teóricas y prácticas de este proceso, dadas las necesidades existentes.

Lo anteriormente expuesto, a partir de los resultados, unido a la experiencia personal de las autoras, y el vínculo laboral con esta problemática permite identificar la existencia de una relación entre la forma en que se concibe y dirige el proceso de formación permanente y los niveles de profesionalidad y desempeño que alcanzan los bibliotecarios. Estos argumentos permiten establecer como inconveniente insuficiencias en la preparación profesional del bibliotecario universitario que limitan su desempeño para enfrentar los cambios imperantes de las universidades cubanas.

En la búsqueda de alternativas de solución para revertir la situación descrita, se revela la necesidad de fundamentar una propuesta teórica y práctica asociada a la formación permanente de los bibliotecarios, a partir de comprender las particularidades, necesidades, limitaciones, ventajas e intereses.

Esta intencionalidad precisó de la consulta y análisis de documentos e investigaciones acerca del objeto de estudio en los que se plantean: estrategias, programas, sistemas o modelos de formación, capacitación, perfeccionamiento, tales como el estudio realizado por (Valiente, P., 2001), (Fuenmayor, 2011), (Carvajal, 2014), no obstante, es indispensable indagar y dar Volumen 1 | número 2 | julio-diciembre | 2018 
respuestas a las necesidades de formación de los bibliotecarios a través de un redimensionamiento de su desempeño en los contextos actuales.

En correspondencia con ello, se ha precisado como objetivo la elaboración de una estrategia para la capacitación técnico-pedagógica para los bibliotecarios de la Universidad de Oriente, sustentada en un modelo de capacitación que connota la relación dialéctica entre los contenidos técnicos de la bibliotecología y los contenidos pedagógicos; temática de gran actualidad determinada por la modelación realizada de la capacitación técnico-pedagógica con salida en la estrategia de capacitación técnico pedagógica y en correspondencia con la política actual del Estado cubano, el MINED, el MES y los objetivos estratégicos; insertado a los objetivos y tareas del proyecto "Nodos formativos y desempeño exitoso del profesional de la educación en contextos educativos".

\section{DESARROLLO}

A partir de las transformaciones que ocurren en el sistema de educación superior, es evidente la apremiante necesidad de cambiar algunas cuestiones en cuanto a la formación de los bibliotecarios en las universidades; de modo que se conviertan en agentes transformadores y dinamizadores de la educación para la vida, a partir del rol que deben desempeñar en los diferentes contextos de actuación.

Esto confirma la idea de que debe existir relación entre formación inicial y permanente de los mismos, idea que encuentra sus fundamentos en las concepciones actuales sobre la educación permanente comprendida en todas sus dimensiones como principio que caracteriza la Educación del Siglo XXI.

Desde épocas remotas pensadores como Comenio (1983), defendieron la idea de que la educación fuera un proceso sin limitaciones en el tiempo y en el espacio y señaló explícitamente en su Didáctica Magna... a los hombres se les ha dado la oportunidad para la perfectibilidad continua e interminable, para la creatividad, para la educación permanente y para la autoeducación. Todo el mundo tiene el deber de aprovechar esta oportunidad, en otro momento agrega...el hombre ha de convertirse en un todo armónico si han de desarrollarse plenamente todas sus potencialidades y habilidades. Estas ideas tienen plena validez, pues entre las múltiples funciones de la educación y la formación del individuo se encuentra el aprendizaje sistemático, que permite a los sujetos adaptarse a las incesantes transformaciones del mundo laboral y a la extensión y obsolescencia del conocimiento.

En la actualidad se hace énfasis en el concepto de educación a lo largo de toda la vida, como la llave para entrar en el Siglo XXI y se afirma según Delors (1996), que toda ocasión debe ser aprovechada para aprender y desarrollar capacidades por parte del sujeto. En tal sentido, la formación es un proceso que acompaña de manera consciente al ser humano durante toda su vida en relación con el resto de la sociedad, con el contexto y con la historia donde las tradiciones socioculturales, los intereses políticos ideológicos encuentran el camino de la transmisión. Se considera necesario comprender que el proceso de formación se vincula con los objetivos y tareas que emanan de las necesidades sociales concretas, en relación con el medio que le rodea.

Baxter (2003) plantea que en la Pedagogía Cubana, la formación del hombre se concibe, como el resultado de un conjunto de actividades organizadas de modo sistemático y coherente, que le permiten poder actuar consciente y creadoramente. Este sistema debe prepararlo como sujeto activo de su propio aprendizaje y desarrollo; hacerlo capaz de transformar el mundo en que vive y transformarse así mismo; formar al hombre es prepararlo para vivir en la etapa histórica concreta en que se desarrolla su vida.

En esta misma línea de pensamiento Chávez y Lemus (2015), consideran que la formación como una función de la educación: (...) es un proceso que tiene como medio a la instrucción y a la educación (...) y que orienta al desarrollo (p. 4), del mismo modo López Hurtado (2012) la concibe como la orientación del desarrollo hacia el logro de los objetivos de la educación, como expresión de la dirección de ese desarrollo.

En este mismo sentido, García y Addine (1997) son del criterio que la formación actual del docente es un proceso de profesionalización, por su rápida inserción en la práctica.

A partir de estas ideas, para el análisis general de las concepciones pedagógicas en el proceso de formación de los profesionales, se asume lo declarado por Horruitinier (2007) acerca de las tres dimensiones que él identifica en el proceso formativo: la dimensión instructiva que en este estudio permite poner al bibliotecario en contacto con el objeto de su profesión, considerando no solo el contenido como conocimiento sino también 
el desarrollo de habilidades profesionales, valores expresados en la conducta, modos de actuación, actitudes que regulan su comportamiento para que sean hombres útiles socialmente, de ahí, se es consecuente además con el autor antes referido al valorar la vinculación de estas tres dimensiones en la investigación que se desarrolla.

En síntesis, lo educativo persigue la formación para la vida; lo instructivo, la formación como trabajador, como ser social heredero de una cultura que le permita desempeñarse profesional y culturalmente en la vida, y lo desarrollador, la formación de capacidades, valores éticos y morales, las cuales se integren de manera holística para propiciar la formación integral del profesional en cuestión de manera sistemática.

Por su parte Flor y Alba (2011); señalan que la formación permanente tiene su esencia en la profundización, ampliación y actualización en su preparación profesional, mayor dominio del contenido de la profesión, y en el necesario perfeccionamiento de las competencias profesionales y capacidades para la investigación e innovación, teniendo en cuenta su continuo crecimiento personal y desarrollo humano.

La definición anterior, aunque tiene un carácter generalizador y no se refiere directamente a la formación docente o de bibliotecarios, expresa aspectos puntuales del proceso que permite la preparación para la vida del individuo y el dominio de la profesión y explicita un aspecto que, para los fines de la investigación tiene un connotado valor, que es precisamente la contribución de la formación permanente al crecimiento personal y profesional. Al analizar estos argumentos se demuestra la importancia que tiene en la actualidad la formación permanente como vía para mejorar y perfeccionar el desempeño de los bibliotecarios a partir de las funciones que demanda el contexto educativo donde interactúan.

En este sentido, se connota su vinculación ya que actúan en las relaciones del hombre con la sociedad, de gran importancia y permiten comprender la actividad valorativa y el papel que desempeñan las necesidades y los intereses en la propuesta con fines para alcanzar el mejoramiento del desempeño profesional del bibliotecario universitario, asumiendo una dinámica de formación permanente, en correspondencia con las tendencias de cambios acelerados en el campo científico - tecnológico y, específicamente, pedagógico y didáctico.

La formación permanente tiene su primer fundamento en la filosofía marxista - leninista, a través del aporte que le hace el método general dialéctico - materialista que le sirve de base, el cual permite alejarse del dogmatismo y de visiones unilaterales de ella como proceso y analizarlo en el decursar de su historia y de forma dialéctica en función del contexto histórico concreto en que ahora se desarrolla.

A partir de la teoría dialéctico materialista de Marx, la pedagogía, la psicología y la sociología educativa, se reconoce el carácter dialéctico de la relación entre el sujeto y la sociedad, y de esta con la educación, donde las influencias son recíprocas y cambiantes.

En tal sentido, Buonocuore (1976) sostenía, que la enseñanza de la bibliotecología debe adecuarse a las condiciones históricas actuales y a las necesidades efectivas y concretas de cada tipo de usuario. De lo que se infiere que el bibliotecario debe tener una mente ágil y viva capaz de transformar y ser capaz de captar los acontecimientos más novedosos e inesperados...

Por su parte, Carro (1997) plantea que: en esta variedad de tecnologías, servicios y productos de información, las bibliotecas deberán orientarse adecuadamente para satisfacer cada necesidad con la mejor opción a su alcance. Para ello, los bibliotecarios requieren de una preparación y cultura mínima sobre las ofertas disponibles...

El especialista Alburquerque (1998, p. 7-8) es de la opinión que [...] el profesional de este campo del saber se encuentra como en un punto entre el pasado y el futuro. Convive con tareas y técnicas tradicionales de su profesión, pero precisa atravesar rumbo a otra realidad, donde están situados sus clientes y aprender a convivir con lo nuevo y lo inusitado, en una constante renovación de la novedad $(. .$.

Estudios preliminares de diversos especialistas de la bibliotecología y las ciencias de la información han incursionado en el tema que se estudia con las propuestas de talleres, acciones y actividades metodológicas que propicien el mejoramiento del desempeño de los bibliotecarios, algunas apuntan hacia el procesamiento de la información, otras al trabajo con el currículo de la biblioteca y a la promoción de lectura, sin embargo, resultan escasas las propuestas de vías, alternativas, estrategias, modelos, entre otras, para dar solución al limitado desarrollo de la capacitación técnico-pedagógico de los bibliotecarios. Es decir, propuestas teórico-prácticas, que tengan en cuenta la formación y capacitación con carácter permanente en el aspecto técnico relacionado con contenidos específicos de la bibliotecología y las

Volumen 1 | número 2 | julio-diciembre | 2018 
ciencias pedagógicas, que promueva una óptima preparación y desempeño de los bibliotecarios.

Tradicionalmente la biblioteca como institución ha tenido un lugar importante en el desarrollo cultural de la sociedad, desde diferentes aristas del saber humano. A partir del año 1959, comienza un nuevo período en la vida del pueblo cubano, lo cual tuvo, una repercusión en el ámbito político, económico y social, particularmente en la educación, en la que se promueve una política educativa donde la formación y superación constante de los profesionales ocupa un lugar privilegiado, otro hecho importante acaecido, lo constituye la creación del departamento nacional de bibliotecas e inicio de su formación regular, la creación y fortalecimiento del SIED y el diseño e implementación de la concepción estratégica de este. Fundamentos que la autora de esta investigación consideró para seleccionar las siguientes etapas de su periodización.

$\checkmark$ 1ra etapa, 1960 - 1984. Se caracterizó por la creación de bibliotecas y la organización de la actividad científico informativo, así como, el inicio de la formación regular de los bibliotecarios través de cursos presenciales desde un enfoque tradicional.

$\checkmark$ 2da etapa, 1985 - 1999.) fue de nuevas perspectivas de formación y capacitación de los bibliotecarios, a partir de la creación y fortalecimiento del Sistema de Información para la Educación, (SIED).

\section{$\checkmark$ 3ra etapa, 2000 - 2018) las} concepciones han ido evolucionado significativamente, a partir de los cambios ocurridos en todos los subsistemas del MINED y el MES de manera positiva. Se aprueba un nuevo plan de estudios para la Enseñanza Técnico Profesional, sin embargo, aún no se aprecia, ni se consolida de manera estable y coherente la formación y capacitación integral que favorezca la conciliación de los contenidos técnicos de bibliotecología y con los contenidos de carácter pedagógico de los bibliotecarios sustentado en un modelo lógico que propicie el éxito del buen desempeño de una biblioteca universitaria.

El análisis anterior permite revelar como tendencias fundamentales a través de los tránsitos:
- Desde una orientación de las actividades de formación permanente con un enfoque tradicional y una concepción normativa caracterizada por temáticas generales sobre la dirección y organización de la biblioteca a una orientación de formación relacionada con la labor técnica del bibliotecario.

- Desde una visión tradicional de la formación permanente del bibliotecario a una perspectiva de profesionalización y capacitación contextualizada a sus necesidades concretas, todavía insuficiente ante las limitaciones que posee este profesional en los contenidos de las ciencias técnicas y pedagógicas para su desempeño eficiente.

Desde esta perspectiva el modelo pedagógico que se propone parte de un enfoque materialistadialéctico y se fundamenta en los referentes del enfoque histórico-cultural de Vigotski, L. y sus seguidores que, al explicar la relación entre educación y desarrollo, sostiene que la educación precede al desarrollo, lo impulsa; pero debe tener en cuenta el desarrollo alcanzado, lo que quiere decir que no solo incluye el desarrollo actual sino también, la zona de desarrollo próximo.

Se define como Modelo de capacitación técnicopedagógica de los bibliotecarios, a la construcción teórica que fundamenta científicamente la capacitación, actualización y perfeccionamiento de este profesional en servicio a partir del sistema de relaciones que se establecen entre los componentes de los subsistemas que lo conforman en la que se integran los aspectos técnicos específicos de la bibliotecología y los contenidos pedagógicos necesarios para el desarrollo de cualidades profesionales y humanas que les permita la orientación, proyección y control educativo a los miembros de la comunidad de usuarios (estudiantes, docentes, investigadores, y otros miembros de la comunidad socio-cultural), así como trasferir los conocimientos adquiridos a cualquier contexto de aprendizaje. El modelo pedagógico que se construye presenta, subsistemas, componentes, funciones y cualidades que están en consonancia con el método sistémico estructural y los presupuestos teóricos anteriormente declarados.

Dicho modelo, visto como sistema, consta de dos subsistemas que funcionan en interrelación dialéctica, ellos son:

\section{EI subsistema de orientación cognitivo motivacional de la}

Volumen 1 | número 2 | julio-diciembre | 2018 
capacitación técnico-pedagógica de los bibliotecarios escolares. Este subsistema da cuenta de la relación de interdependencia que se establece entre los componentes a) la actividad técnico informativa, b) la motivación profesional, c) el perfil de idoneidad en la que uno aporta al otro, a partir de los contenidos, recursos, capacidades y habilidades. De esta relación emerge como cualidad resultante la orientación contextualizada de la capacitación técnico pedagógica. Precisa el encargo social a la labor profesional de los bibliotecarios, expresado en el desempeño profesional, los valores éticos y estéticos, la comunicación asertiva, así como las funciones y roles de su actividad técnico informativa. De las relaciones entre los componentes del subsistema de orientación cognitivo motivacional, a partir de las funciones que ejercen, emerge como nueva cualidad de nivel superior: la orientación contextualizada de la capacitación técnico pedagógico del bibliotecario escolar.

2. El subsistema proyectivo regulador de la capacitación técnico-pedagógica de los bibliotecarios es el que define la planeación, dinamización y evaluación del proceso con un enfoque estratégico. Comprende tres procesos esenciales: a). diseño de la proyección de la capacitación técnico-pedagógica, b) aprehensión y transposición del contenido de la capacitación técnico-pedagógica y c) evaluación y retroalimentación de la capacitación técnico-pedagógica. Por lo que reconoce una actividad constructiva desde el trabajo colaborativo realizada por el bibliotecario y promovida / orientada por el profesor capacitador y que potencian el desarrollo intelectual, del bibliotecario desde lo individual y lo colectivo. De ahí que, de las relaciones entre los componentes del subsistema proyectivo regulador, desde las funciones que le corresponden cumplir, emerge como cualidad esencial: el diseño y realización contextualizada de la capacitación técnico-pedagógica.

De las relaciones que se producen entre los dos subsistemas, emerge la cualidad esencial que caracteriza al sistema: la capacitación técnico pedagógica contextualizada, entendida como la cualidad que expresa la correspondencia de la orientación, diseño, realización y evaluación de la capacitación técnico pedagógica de los bibliotecarios.
La estrategia de capacitación propuesta se caracteriza por la estrecha relación que establece entre las diferentes etapas que la componen reforzando su carácter sistémico.

El término estrategia ha sido abordado por diversos autores, sin embargo, para los fines de esta investigación se asume la definición de estrategia dada por Sierra (2008) quien plantea que estrategia es la concepción teórico- práctica de la dirección del proceso pedagógico durante la transformación del estado real al estado deseado, en la formación y desarrollo de la personalidad, de los sujetos de la educación, que condiciona el sistema de acciones para alcanzar los objetivos, tanto en lo personal, lo grupal, como en la institución escolar. Asimismo, revela el carácter transformador de este instrumental y connota objetivos y acciones para llevar a vías de hecho tal transformación.

\section{Estructura lógica-metodológica de la estrategia de capacitación técnico-pedagógica del bibliotecario.}

Objetivo general de la estrategia: Ofrecer herramientas técnicas metodológicas y pedagógicas que contribuyan a la capacitación técnicopedagógica de los bibliotecarios y a su desempeño profesional.

Esta estrategia está estructurada en tres etapas:

1. Etapa de diagnóstico y sensibilización

2. Etapa de planificación y ejecución

3. Etapa de control y evaluación.

\section{Etapa de diagnóstico y sensibilización:}

Objetivo: Determinar las carencias y potencialidades de los bibliotecarios a través de un diagnóstico participativo que favorezca su sensibilización.

Acciones del profesor capacitador:

- Determinar fortalezas y debilidades de su accionar técnico pedagógico.

- Diseñar el sistema de acciones, instrumentos de diagnóstico y actividades de manera que promueva la reflexión metacognitiva en los bibliotecarios y potencie el tránsito del proceso con enfoque desarrollador, cognitivo y socio-cultural.

- Diagnosticar las carencias y potencialidades cognitivas y afectivo de los bibliotecarios para enfrentar el proceso de capacitación, a través de pruebas pedagógicas.

Volumen 1 | número 2 | julio-diciembre | 2018 
- Intercambiar los resultados del diagnóstico con los bibliotecarios participantes para propiciar la reflexión acerca de estos.

Acciones del bibliotecario escolar:

- Constituirse en un ente activo que participa en el proceso de diagnóstico, sensibilizándose con las transformaciones y los resultados esperados.

- Reflexionar y comprender su influencia en el proceso, limitaciones y logros cognitivoafectivos.

- Manifestar su motivación a los profesores capacitadores acerca de la importancia y necesidad de la utilización del trabajo cooperativo en el proceso que se investiga.

- Aportar y compartir ideas, sugerencias extraídas de su práctica técnico pedagógica, que se incorporarán a los saberes del grupo, a partir del compromiso con la tarea.

- Asumir un rol protagónico a través de las técnicas las necesidades formativas, intereses y motivaciones de los mismos.

Las actividades realizadas en esta etapa inicial, permitieron a los sujetos involucrados en la capacitación, diagnosticar las particularidades reales del proceso, al tener en cuenta las necesidades formativas, intereses y motivaciones de los mismos, hacia las técnicas básicas que debe dominar un bibliotecario.

\section{Etapa de planificación y ejecución:}

Objetivos:

- Diseñar las acciones de capacitación en coherencia con las necesidades detectadas sobre los contenidos conceptuales, procedimentales y actitudinales de las técnicas a utilizar por los bibliotecarios desde una perspectiva técnico-pedagógica

- Propiciar la transmisión y aprehensión de los contenidos conceptuales, procedimentales y actitudinales relativos a las técnicas fundamentales.

La estrategia de capacitación centra su atención en las acciones esenciales que permitirán llevar a vías de hecho su etapa de ejecución. Estas acciones son las siguientes:

- Seleccionar en interacción con los bibliotecarios la modalidad y la vía de concretarse.

- Prever la disponibilidad de medios, locales y personal para el desarrollo de la capacitación.
- Determinar los objetivos que guiarán el proceso de enseñanza- aprendizaje en la capacitación.

- Determinar el sistema de habilidades a desarrollar a través de la capacitación que potencie el perfil de idoneidad del bibliotecario.

- Seleccionar los contenidos a impartir en los cursos que favorezcan su transposición a contextos educativos y comunitarios

- Elaborar los programas y orientaciones metodológicas de los contenidos a impartir.

- Planificar las formas de organización de los cursos desde el trabajo colaborativo.

- Proyectar la planificación, orientación ejecución y evaluación desde tareas metacognitivas que propicien el intercambio, la reflexión y la toma de decisiones para resolver problemas concretos de su práctica.

- Impartir el contenido seleccionado a través del trabajo colaborativo y en interacción con las nuevas tecnologías.

- Orientar y realización, desde las clases prácticas, de diagnóstico integral de la comunidad de usuarios y el contexto sociocultural en que se inserta la biblioteca.

- Orientar actividades a través del método ilustrativo, explicativo para la promoción de lectura, así como las técnicas y procedimientos para la catalogación, clasificación, indización, desde actividades prácticas, como expresión de la actividad técnico-informativa.

- Realizar actividades coordinadas de educación de usuarios a través de la biblioteca universitaria, para propiciar la transposición de lo aprendido, el intercambio, la aprehensión y comprensión de los nuevos roles.

- Aplicar un sistema de tareas y guías que favorezcan la metacognición e interaprendizaje en el grupo.

La estrategia de capacitación que se propone, centra su atención en aspectos esenciales para lograr transformaciones en la actividad que desempeñan los bibliotecarios en la universidad; es por ello que prepararlos en los planos cognoscitivo, procedimental y actitudinal con relación a las técnicas propias del trabajo en las bibliotecas, dados en un espacio de intercambio de experiencia de los participantes en los cursos, favorecerá la preparación técnico pedagógica para el ejercicio de las funciones a desarrollar en la biblioteca universitaria. Desde esta perspectiva, se considera pertinente la propuesta de las siguientes acciones: Un sistema de cursos de capacitación técnico-pedagógico que contribuya a la preparación y actualización cognoscitiva de los Volumen 1 | número 2 | julio-diciembre | 2018 
bibliotecarios en torno a las técnicas de la bibliotecología y contenidos de la pedagogía, que puedan conciliarse en función de elevar la calidad del proceso de enseñanza aprendizaje y docente educativo de modo que contribuya al buen desempeño de este profesional.

\section{Etapa de control y evaluación:}

Esta etapa tiene como objetivo valorar la efectividad de las acciones diseñadas e instrumentadas y constituye un momento importante dentro del proceso ya que los bibliotecarios en trabajo grupal se retroalimentan con las sugerencias y señalamientos que fueron realizados por sus compañeros y capacitador rehace el trabajo, intercambia ideas, juicios con sus compañeros. Luego socializa este a través del trabajo por equipos. Los profesores capacitadores de cada curso deben propiciar la autoevaluación y la coevaluación. Se sugiere llevar un registro de todo lo que acontece en el grupo durante el proceso de capacitación para valorar el crecimiento personal y profesional de los participantes, considerando de esta manera tanto lo afectivo como lo cognitivo.

Para la corroboración de los resultados sobre la aplicación de la estrategia de capacitación sustentada en un modelo de igual naturaleza se establecen los siguientes indicadores:

$\checkmark$ Estructura y correspondencia del modelo pedagógico con el proceso objeto de estudio

$\checkmark$ Estructura y acciones de la estrategia. Concreción del modelo en ella.

$\checkmark$ Posibilidades de aplicación y generalización práctica. Impacto.

A manera de conclusión se reconoce la coincidencia de los especialistas en cuanto a la pertinencia del modelo y la factibilidad de la estrategia pedagógica. Los que, a su criterio, evidencian calidad en su construcción teóricometodológica, revelan la concreción del modelo en la estrategia propuesta y las posibilidades reales de aplicación y generalización práctica, así como de su impacto.

En tal sentido, significan la correspondencia de las etapas de la estrategia pedagógica del proceso objeto de estudio. De igual modo, reconocen la valía del modelo en su contextualización en las condiciones de la profesionalización desde actividad científico informativa y su respuesta a las carencias de la práctica pedagógica.

Todo ello permitió que los bibliotecarios alcancen una notable preparación que le permite elevar a estadios superiores su actuación en el contexto en que se desempeñan, contribuyendo a perfeccionar 9 la labor que realizan como organizar, registrar, clasificar, catalogar, indizar, extractar y preparar físicamente los documentos.

Se logra que en los planes individuales se diseñen actividades de preparación de forma general con énfasis en la organización y el procesamiento técnico de la información.

Los resultados en la etapa experimental con la aplicación de la estrategia pedagógica en la práctica educativa evidencian las transformaciones operadas en el desarrollo del proceso de capacitación. Notable preferencia por el uso de los servicios de la biblioteca universitaria, esencialmente a través de la web 2.0

$\checkmark$ Disfrute de las actividades de promoción, animación de lectura, concursos tanto en la universidad como en el contexto sociocultural y reconocimiento de la utilidad de los textos de su preferencia.

$\checkmark$ Reconocimiento de la significación de la biblioteca universitaria para el aprendizaje y las potencialidades comunicativas.

Los elementos antes analizados permitieron concluir que la estrategia para la capacitación técnico pedagógica es coherente y viable y por consiguiente factible y pertinente.

\section{CONCLUSIONES}

1. A partir del análisis de los resultados de la fundamentación epistémica del proceso de superación de los bibliotecarios con énfasis en la capacitación, permitió corroborar las insuficiencias en el proceso objeto de estudio.

2. La caracterización de la evolución histórica del proceso de formación posibilitó determinar las tendencias pedagógicas que singularizan el proceso estudiado en la Universidad de Oriente Santiago de Cuba.

3. El modelo de capacitación elaborado da cuenta de las relaciones dialécticas que emergen las que adquieren su máxima expresión en la cualidad resultante capacitación profesional contextualizada, evidenciándose un mayor nivel de esencialidad en la praxiología del proceso que se modela, corroborando la relación indispensable entre la contribución a la teoría y el aporte práctico.

Volumen 1 | número 2 | julio-diciembre | 2018 
4. La implementación parcial de estrategia para la capacitación técnico pedagógica de los bibliotecarios propició un mejor desempeño de estos profesionales en la práctica pedagógica.

5. La corroboración realizada a través del criterio de especialistas, el preexperimento y los talleres de sensibilización y socialización, permitió constatar la pertinencia y factibilidad de los aportes en la práctica educativa, lo cual ofrece una alternativa de solución al problema de la investigación.

\section{REFERENCIAS BIBLIOGRÁFICAS}

Alburquerque, A. (1998). Los tres mundos de la Ciencia de la información. Ciencias de la Información, 3,3-4

Baxter, E. (2003). La escuela y el problema de la formación del hombre. En: Compendio de Pedagogía. (pp.143-192). La Habana: Editorial Pueblo y Educación.

Buonocuore, J. D. (1976). Diccionario de Bibliotecología. Término relativos a la bibliología, bibliografía, bibliofilia, bibliotecología, archivología, documentología, tipografía y materias afines. Buenos Aires: Ediciones Marymar.

Carro, J. R. (1997). La automatización de bibliotecas y las redes locales. Herramientas auxiliares. La Habana: Instituto de Información Científica y Tecnológica (IDICT).

Carvajal, B. (2014). Competencias informacionales desde la formación inicial del docente. Tesis en opción al grado científico de Doctor en Ciencias Pedagógicas. UCP José Martí. Camagüey

Comenio, J. A. (1983). Didáctica Magna. La Habana: Pueblo y Educación.

Chávez, J. y Pérez Lemus, L. (2015). Fundamentos de la Pedagogía General: Parte I. La Habana: Editorial Pueblo y Educación.
Delors, J. (1996). Educar a los protagonistas del futuro. En: Revista Correo de la UNESCO. (24), $16-20$

Flor, G. y Alba, O. (2011). Evaluación del impacto de la formación y capacitación profesional. Quito-Ecuador: Editorial Ecuador F.B.T.

Fuenmayor, M. (2011). La interdisciplinariedad en el proceso de formación permanente de los docentes del PFG en estudios jurídicos de la Universidad Bolivariana de Venezuela. Tesis en opción al grado científico de Doctor en Ciencias Pedagógicas, IPLAC. La Habana.

García, G. y Addine, F. (1997). Formación pedagógica y profesionalización permanente de los docentes. La Habana: Congreso Internacional Pedagogía. IPLAC.

Horruitinier, P. (2007). Fundamentos del proceso de formación en la educación superior: (la experiencia cubana). La Habana: Ministerio de Educación Superior.

López, J. (2012). El carácter científico de la pedagogía en Cuba. La Habana: Editorial Pueblo y Educación.

Sierra, R. A. (2008). La estrategia pedagógica, su diseño e implementación. La Habana: Editorial Pueblo y Educación.

Valiente, P. (2001). La concepción sistémica de la superación de los directores de Secundaria Básica. Tesis en opción al grado científico de Doctor en Ciencias Pedagógicas. ISP José de la Luz y Caballero, Holguín. 